\title{
The role of stellar mass and mass functions in the ISM dust feedback (Research Note)
}

\author{
D. Falceta-Gonçalves
}

\author{
Núcleo de Astrofísica Teórica, CETEC, Universidade Cruzeiro do Sul, Rua Galvão Bueno 868, CEP 01506-000 São Paulo, Brazil \\ e-mail: diego.goncalves@unicsul.br
}

Received 10 May 2007 / Accepted 22 October 2007

\section{ABSTRACT}

\begin{abstract}
Context. The dust component of the interstellar medium (ISM) has been extensively studied in past decades. Late-type stars have been assumed as the main source of dust to the ISM, but recent observations show that supernova remnants may play a role in the ISM dust feedback.

Aims. In this work, we study the importance of low and high mass stars, along with their evolutionary phase, in the ISM dust feedback process. We also determine the changes in the obtained results considering different mass distribution functions and star formation history.

Methods. We describe a semi-empirical calculation of the relative importance of each star at each evolutionary phase in the dust ejection to the ISM. We compared the obtained results for two stellar mass distribution functions, the classic Salpeter initial mass function, and the current mass function. We used the evolutionary track models for each stellar mass and the empirical mass-loss rates and dust-to-gas ratio.

Results. We show that the relative contribution of each stellar mass depends on the used distribution. Ejecta from massive stars represent the most important objects for the ISM dust replenishment using the Salpeter IMF. On the other hand, for the present mass function, low and intermediate mass stars are dominant.

Conclusions. We confirm that late-type giant and supergiant stars dominate the ISM dust feedback in our Galaxy, but this may not the case for galaxies experiencing high star formation rates or at high redshifts. In those cases, SNe are dominant in the dust feedback process.
\end{abstract}

Key words. ISM: dust, extinction - stars: luminosity function, mass function - stars: mass-loss - stars: winds, outflows - evolution

\section{Introduction}

Red giant branch (RGB) and asymptotic giant branch (AGB) stars are known as the major continuous dust producers in the Universe, but also supernova $(\mathrm{SN})$ remnants have shown fast grain growth and dusty shells of $M_{\mathrm{d}} \sim 10^{-2}-10^{1} M_{\odot}$ (Nozawa et al. 2003). From the classical nucleation theory, the timescale for grain growth in the interstellar medium (ISM) can be estimated by $\tau_{\mathrm{g}}=4 s a\left(f n_{i} m_{i} v_{i}\right)^{-1}$, where $a$ is the dust mean size, $s$ the material density, $f$ the sticking probability, $n_{i}$ the gas phase density, $m_{i}$ the atom mass, and $v_{i}$ the velocity of the $i$ th atom to be added onto the grain surface. Considering typical ISM parameters, $a \sim 0.1-1 \mu \mathrm{m}, s \sim 2 \mathrm{~g} \mathrm{~cm}^{-3}, n_{i} \sim 1 \mathrm{~cm}^{-3}, T \sim 10 \mathrm{~K}$, and assuming an efficiency $f=0.1-1$, we find $\tau_{\mathrm{g}}<10^{9} \mathrm{yr}$.

Dust particles are likely to be destroyed by shock waves (Draine \& Salpeter 1979; McKee 1989). Grain-grain or ion-grain collisions will lead to the shattering process, reducing or destroying dust particles. Jones et al. $(1994,1996)$ described the shattering process of the ISM dust induced by SN blasts, obtaining destruction timescales of $\tau_{\mathrm{d}} \sim 4-6 \times 10^{8} \mathrm{yr}$. Actually, as mentioned in these works, accurate derivation of destruction timescales depends on the velocity of the shock waves, the frequency of SNe, and the physical properties of the ISM, such as density and temperature. Hence, the destruction timescales are shorter than the dust growth scale, and the observed dust could not be explained by nucleation in loco, but had to be recently $\left(<10^{9} \mathrm{yr}\right)$ injected into the ISM (Tielens 1998).

It is commonly suggested that the ISM dust should mostly originate from evolved low and intermediate mass stars but recent observations have shown the presence of large quantities of dust $\left(M_{\mathrm{d}}=10^{8} M_{\odot}\right)$ in the early Universe $(z>5)$ (Hughes et al. 1998; Archibald et al. 2001; Dunne et al. 2003; Bertoldi et al. 2003; Maiolino et al. 2004). At that age, low and intermediate mass stars had not evolved yet, so SNe are recognized as responsible for such material ejections (Todini \& Ferrara 2001; Morgan \& Edmunds 2003; Sugerman et al. 2006; Dwek et al. 2007).

In the post-shock phase of the $\mathrm{SN}$ remnant, the gas is generally cool and dense enough to allow dust formation and growth (Falceta-Gonçalves et al. 2003, 2005). Observationally, it is confirmed for SN1987A, which shows a $10^{-3} M_{\odot}$ dust shell with a dust-to-gas ratio for heavier elements of $\sim 0.3$. The same result was obtained for several other galactic and extragalactic $\mathrm{SNe}$ (Barlow et al. 2005; Gomez et al. 2007). Therefore, SNe seem to play an important part on the ISM dust replenishment process (Dwek 1998). However, if the short dust-destruction timescale is taken into account, SNe could only be the main source of dust of the Galaxy if it presented high star formation rates in its recent history.

In this work we present a semi-empirical model, in which we study the role of different stellar mass functions in the output 


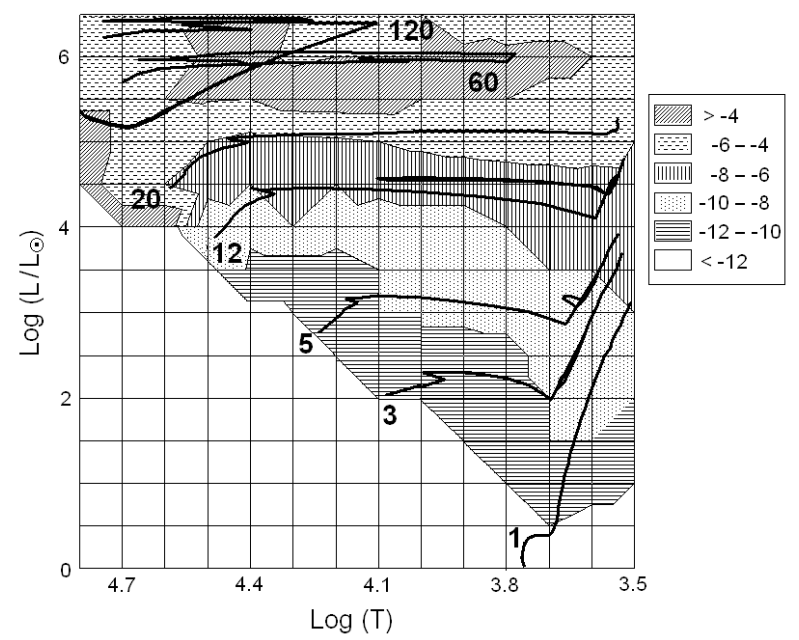

Fig. 1. Evolutionary tracks for stars with masses ranging from 1 to $120 M_{\odot}$ for metallicity of 0.02 . The box shows the values of $\log (\dot{M})$.

of dust ejected into the ISM. The model is described in Sect. 2, while in Sect. 3 we show the main results and present a brief discussion, followed by the conclusions.

\section{The model}

To obtain the total amount of dust ejected by a certain star during its full evolution, we have to integrate the dust mass loss rates of each evolutionary phase over the evolutionary time. The difficulties performing such calculations lie in determining the dust mass loss rates at each evolutionary phase of the star, as well as its duration. To accomplish this we used the evolutionary tracks given by Schaller et al. (1992), which take the mass loss during the stellar evolution into account. These numerical calculations also provided the abundance of heavier elements on the stellar surface at each epoch of stellar evolution. We used these numerical results to determine the amount of material able to be added to the dust grains that is being ejected from the stars.

The total dust mass ejected by each star over its lifetime can be determined by integrating the mass loss rate at each stellar evolutionary phase, determined by

$\int_{M_{1}}^{M_{2}} \int_{t_{1}}^{t_{2}} \Phi(m) \dot{M}_{\mathrm{d}}(m) \mathrm{d} t \mathrm{~d} m$,

where $\Phi(m)$ is the stellar population mass distribution, $\dot{M}_{\mathrm{d}}(m)$ the dust mass loss rate of a given star of mass $m$ at the time $t$, $M_{1}$ and $M_{2}$ are the limits of the mass range, and $t_{1}$ and $t_{2}$ are the limits of the time interval. To compute the dust mass loss rates we used

$\dot{M}_{\mathrm{d}}(t, m)=\dot{M}(t, m) f_{\mathrm{d}}(t, m) \chi(t, m)$,

where $\dot{M}$ is the total mass loss rate, which is obtained empirically as described in the following subsection, $f_{\mathrm{d}}$ is the dustto-gas fraction of elements heavier than $\mathrm{He}$, and $\chi$ is the wind metallicity of a star with mass $m$ at an evolutionary time $t$.

In Fig. 1 we illustrate the evolutionary track given by Schaller et al. (1992) for stars with masses ranging from 0.8 to $120 M_{\odot}$ used in this work, for an initial metallicity of 0.02 , and the empirical stellar mass loss rates.

Different models of homogeneous and heterogeneous condensation theories have been proposed, but the actual dust-to-gas fraction of stellar winds is still not completely understood. The gas acceleration is responsible for the gas rarefaction at the base of the stellar atmosphere and, depending on the model used, may result in very different pressure and temperature profiles, which are critical to the derivation of the condensation rates. As an approximation, we introduce a single dust-to-gas fraction for each stellar mass, depending on the chemical composition. The yields for the dust-to-metal fraction $\left(f_{\mathrm{d}}\right)$ for the massive stars were the same used by Dwek et al. (2007) (Table 2), and for low and intermediate mass stars, we used the data from Morgan \& Edmunds (2003) (Table 1).

\subsection{The stellar mass-loss rates}

The mass loss rates for each stellar mass at each evolutionary phase were introduced empirically. For low and intermediate mass stars during the main sequence, giant, and supergiant evolutionary phases, we used the data from de Jager et al. (1988). The wind velocities for giant and supergiant stars are, in general, $v<200 \mathrm{~km} \mathrm{~s}^{-1}$, and dust is not destroyed at heliopause. At the end of their lives, intermediate mass-stars present very high mass loss rates in the post-AGB's phases $\left(\sim 10^{-4} M_{\odot} \mathrm{yr}^{-1}\right)$ (Wachter et al. 2002), and are known as progenitors of the planetary nebulae.

Massive stars are also known to present dust in their winds, mainly in the later stages of their evolution. The WR and LBV stars show high mass loss rates in unstable and clumpy winds that favor dust nucleation and survival. For these objects we used the data given by Crowther (1997). Also, about $30 \%$ of these objects are in binary systems, and Marchenko et al. (2002) find that binary systems present high dust-production rates due to the wind-wind shock, with 4-6\% of the wind mass condensed into grains in $\mathrm{WR}+\mathrm{OB}$ systems. On the other limit, low mass protostars were also considered since their jets and disks are dust growth sites. Typically, these objects have a mass-loss rate of $10^{-8} M_{\odot} \mathrm{yr}^{-1}$ during $10^{6}-10^{7} \mathrm{yr}$ (Mundt et al. 1987).

\subsection{The stellar mass distributions}

To compute the total contribution to the ejected material by all stars, we may define a mass distribution function. To simulate the distribution of a young stellar population, we used the typical Salpeter single-component IMF (Salpeter 1955):

$\Psi(\log M)=A M^{-x}$

for $M_{*}>1 M_{\odot}$, where $A=4.43 \times 10^{-2}$ and $x=1.3$, and

$\Psi(\log M)=B \exp \left[-\left(\log M-\log M_{c}\right)^{2} / 2 \sigma^{2}\right]$

for $M_{*}<1 M_{\odot}$, where $B=0.158, M_{c}=0.079$, and $\sigma=0.69$. For the old populations, which exhibit a significantly lower number of massive stars, we used the present-day mass function (PDMF). For the PDMF, Eqs. (3) and (4) are still valid, but $A=4.4 \times 10^{-2}$ and $x=4.37$ for $0 \leq \log M \leq 0.54, A=1.5 \times 10^{-2}$ and $x=3.53$ for $0.54<\log M \leq 1.26$ and $A=2.5 \times 10^{-2}$ and $x=2.11$ for $\log M>1.26$ (Chabrier 2003; Elmegreen 2004).

\section{Results and discussion}

If one assumes that the dust is rapidly destroyed in the ISM $\left(\tau_{\mathrm{d}} \sim 10^{8} \mathrm{yr}\right)$, it is possible to naively separate the possible scenarios of the galactic history in two: $i$ - old feedback process, which occurred in the presence of a large number of massive stars, and $i i$ - recent feedback process, with a relatively larger 


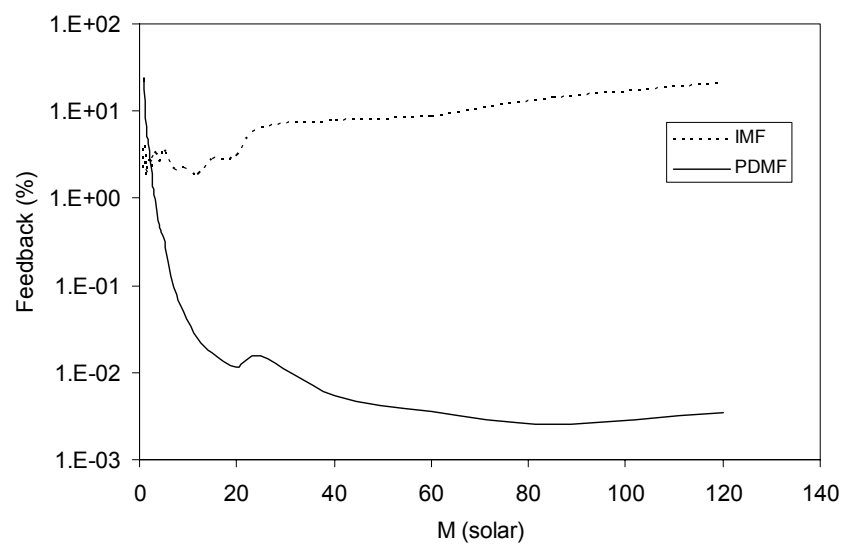

Fig. 2. Dust mass, relative to total (in percent), ejected to the ISM by each stellar mass range during its complete evolution. The solid line represents the model using a typical Salpeter IMF, and the dotted line represents the model for the present-day mass function (PDMF).

number of low and intermediate mass stars. Therefore, we must study the dependence of different stellar mass distributions on the dust feedback process. We performed the calculations of Eqs. (1) and (2) by computing the ejected material during the evolution of all stars using the IMF and PDMF distributions.

In Fig. 2, we show the relative amount of dust ejected by each stellar mass range to the ISM during all its evolution, for the IMF and the PDMF. It is noticeable that the high mass component is the major source of dust for the Salpeter IMF distribution. These stars evolve rapidly and eject large amounts of heavy atoms during the SN phase, and their remnants evolve to form shells of a few solar masses of dust particles. On the other hand, the result is the opposite if we use the present stellar distribution of the Galaxy. There are a few high mass stars so that $\mathrm{SNe}$ are not very frequent. Using the PDMF, the low and intermediate mass stars, as they leave the main sequence, are the main contributors of dust to the ISM. Intermediate mass stars at the supergiant phase were assumed to be the most important source of dust but, surprisingly, the results for the PDMF show that stars with $M_{*}<3 M_{\odot}$ dominate. It can be explained by the overabundance of low mass stars in this distribution.

In the present stage of the Galaxy, the PDMF would give more accurate abundances of dust in the ISM, as the dust is destroyed, or severely changed, on short timescales $\left(\tau_{\mathrm{d}} \sim 10^{8} \mathrm{yr}\right)$. Since the dust generated from high mass stars in the past was recycled, and because these objects are absent in the current population, we may conclude that low mass stars are the main source of the dust observed in the present stage of the Galaxy.

In Table 1 we show the quantitative results of the calculations described above. For a typical IMF, the high mass stars $\left(M_{*}>\right.$ $\left.8 M_{\odot}\right)$ are responsible for $68 \%$ of the dust that returns to the ISM. On the other hand, considering a PDMF, this contribution falls to less than $1 \%$. To determine the absolute feedback mass, we used a total stellar population of $1 \times 10^{10} M_{\odot}$, which gives a total dust mass $M_{\mathrm{d}} \sim 7 \times 10^{7}$ for the IMF distribution and $2 \times 10^{7}$ for the PDMF.

Interestingly, the results indicate that in recent starburst regions, one should expect a higher dust-to-gas ratio when compared to an evolved population. It could possibly be the reason for irregular and spiral galaxies, which present high star formation activity and have more dust than the evolved elliptical galaxies (Seaquist et al. 2004). However, since we have very different scenarios here, with different feedback and destruction
Table 1. Relative and absolute dust ISM feedback contribution for different stellar component mass functions.

\begin{tabular}{ccc}
\hline \hline Stellar mass range & IMF & PDMF \\
\hline$M_{\mathrm{d}}\left(M>8 M_{\odot}\right)$ & $68 \%$ & $1 \%$ \\
$M_{\mathrm{d}}\left(M<8 M_{\odot}\right)$ & $32 \%$ & $99 \%$ \\
$M_{\text {d_total }}{ }^{a}\left(M_{\odot}\right)$ & $7 \times 10^{7}$ & $2 \times 10^{7}$ \\
\hline
\end{tabular}

${ }^{a}$ Assuming a total stellar population of $1 \times 10^{10} M_{\odot}$.
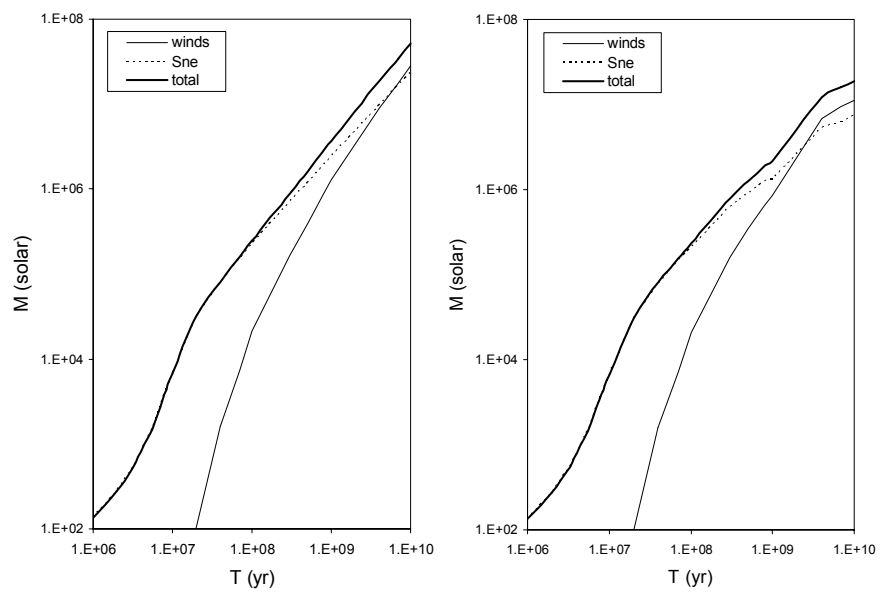

Fig. 3. Absolute dust mass ejected to the ISM. The modeled ejection by high mass stars (solid thin), low and intermediate mass stars (dashed), and total (solid thick), without dust destruction (left panel) and considering dust destruction (right panel).

timescales for each type of galaxy, this statement needs to be tested by more detailed calculations.

\subsection{Time evolution}

In the previous calculations, to obtain the total amount of dust ejected to the ISM, we had to assume the mass function of the stellar population, which translates the evolutionary phase of the stellar component at the epoch we are studying as the dust is recycled in short timescales. However, to obtain the time evolution of the ISM dust component, we have to include the star formation rate function over history in Eq. (1).

We performed the evolutionary calculation assuming a constant star formation rate of $5 M_{\odot} \mathrm{yr}^{-1}$, as used by Morgan \& Edmunds (2003). However, unlike that work, we took the dust destruction into account and studied its role on the total dust ejected into the ISM. At each time step, we calculated the total ejection from each stellar mass range of the current population, added new stars using the Salpeter IMF, removed stars that have already evolved and, finally removed the destroyed dust from the total solid component. In the present calculations, to simulate the chemical enrichment of the stars, a metallicity of $z=0.01 z_{\odot}$ was arbitrarily used for the initial population $\left(t<10^{7} \mathrm{yr}\right)$, and $z=z_{\odot}$ for $t>10^{6}$ yr. In Fig. 3 we show the results for the absolute dust mass ejected into the ISM.

In both cases we can identify the dominance of massive stars in the total ejections at the earlier stages of the galaxy evolution. In both models, the appearance of substantial dust amount from low and intermediate mass stars is only noticeable after $\sim 10^{8}$ yr. These objects become the major dust producers in the last 0.5 billion years on both models. The main differences appear in the later stages, where considering dust destruction, massive stars would be responsible for 3 times less 
dust mass than obtained by previous calculations, while this proportion for low and intermediate mass stars is negligible. After 10 billion years of evolution, we obtained a current dust mass of $\sim 6 \times 10^{7} M_{\odot}$ without dust destruction, and $\sim 10^{7} M_{\odot}$ when taking the dust destruction into account.

\section{Conclusions}

It is still unclear what the main source is of the dust feedback in our Galaxy. The dust must be formed in stars and ejected into the ISM, and some authors have argued for cool late-type stellar winds, which present high mass-loss rates, and observations prove that dust is formed in these sites. On the other hand, other plausible sources are the SNe ejecta. During the final evolutionary phases, high mass stars explode and supersonically eject a very rich gas into the ISM.

First, to determine the relative amount of dust ejected into the ISM for each stellar mass at each evolutionary phase, we calculated the dust ejection during each evolutionary phase of the stars for different stellar mass distributions. As a main result, we showed that $\mathrm{SNe}$ are the main source of dust feedback if a classic Salpeter IMF distribution is assumed. On the other hand, if we use the current mass function, we show that the main sources of dust to the present Galaxy are the low and intermediate mass stars, representing more than $90 \%$ of the total dust mass.

Second, we studied the dust feedback process along the galactic time evolution, as done by Morgan \& Edmunds (2003), but including the effects of dust destruction by SN blasts. For simplicity we used a constant destruction rate, consistent with the current galactic physical parameters. During previous ages, the $\mathrm{SNe}$ ejecta were dominant, in agreement with previous works. We showed that, considering the dust destruction, low and intermediate mass stars are dominant for a galactic age of $t>10^{9} \mathrm{yr}$, in a much higher proportion. The total dust mass of $\sim 10^{7} M_{\odot}$ is obtained for a star formation rate of $5 M_{\odot} \mathrm{yr}^{-1}$.

The dust destruction timescale depends on the $\mathrm{SNe}$ frequency, as well as on the ISM density and temperature. It is probable that the destruction rate was higher earlier in the galactic evolution. In this case, the presented conclusions will stand, and the role of low and intermediate mass stars in later stages will be even higher.

Acknowledgements. D.F.G thanks FAPESP (No. 06/57824-1) for financial support, also A. Jones and the referee for the comments and suggestions.

\section{References}

Archibald, E. N., Dunlop, J., Hughes, D. H., et al. 2001, MNRAS, 323, 417 Barlow, M. J., Sugerman, B. E. K., Fabbri, J., et al. 2005, ApJ, 627, 113

Bertoldi, F., Carilli, C., Cox, P., et al. 2003, A\&A, 406, 55

Chabrier, G. 2003, PASP, 115, 763

Crowther, P. A. 1997, MNRAS, 290, 59

de Jager, C., Nieuwenhuijzen, H., \& van der Hucht, K. 1988, A\&AS, 72, 259

Dunne, L., Eales, S. A., \& Edmunds, M. G. 2003, MNRAS, 341, 589

Draine, B., \& Salpeter, E. 1979, ApJ, 231, 77

Dwek, E. 1998, ApJ, 501, 643

Dwek, E., Galliano, F., \& Jones, A. 2007, ApJ, 662, 927

Elmegreen, B. G. 2004, MNRAS, 354, 367

Falceta-Gonçalves, D., Jatenco-Pereira, V., \& Abraham, Z. 2003, RMxAA, 15, 71

Falceta-Gonçalves, D., Jatenco-Pereira, V., \& Abraham, Z. 2005, MNRAS, 357, 895

Gomez, H. L., Eales, S. A., \& Dunne, L. 2007, IJAsB, 6, 159

Hughes, D. H., Serjeant, S., Dunlop, J., et al. 1998, Nature, 394, 241

Jones, A. P., Tielens, A. G., Hollenbach, D. J., \& McKee, C. F. 1994, ApJ, 433, 797

Jones, A. P., Tielens, A. G., \& Hollenbach, D. J. 1996, ApJ, 469, 740

Maiolino, R., Schneider, R., Oliva, E., et al. 2004, Nature, 431, 533

Marchenko, S., Moffat, A., Vacca, W., et al. 2002, ApJ, 565, 59

McKee, C. F. 1989, in Interstellar Dust, ed. L. J. Allamandola, \& A. G. G. M.

Tielens (Dordrecht: Kluwer), IAU Symp., 135, 431

Morgan, H. L., \& Edmunds, M. G. 2003, MNRAS, 343, 427

Mundt, R., Brugel, E. W., \& Buehrke, T. 1987, ApJ, 319, 275

Nozawa, T., Kozasa, T., Umeda, H., et al. 2003, ApJ, 598, 785

Salpeter, E. E. 1955, ApJ, 121, 161

Schaller, G., Schaerer, D., Meynet, G., \& Maeder, A. 1992, A\&AS, 96, 269

Seaquist, E., Yao, L., Dunne, L., \& Cameron, H. 2004, MNRAS, 349, 1428

Sugerman, B. E. K., Ercolano, B., Barlow, M. J., et al. 2006, Science, 313, 196

Tielens, A. G. 1998, ApJ, 499, 267

Todini, P., \& Ferrara, A. 2001, MNRAS, 325, 726

Wachter, A., Schroder, K., Winters, J., et al. 2002, A\&A, 384, 452 